\title{
A Survey on Routing Protocols for Vehicular Ad-Hoc Networks
}

\author{
Bouchra Marzak ${ }^{1 *}$, Hicham Toumi', Kamal El Guemmat' ${ }^{1}$ Al Habib Benlahmar² and Mohamed Talea' \\ 'Department of Physical, Hassan II University, Casablanca, Morocco; \\ marzak8bouchra@gmail.com, toumi.doc@gmail.com, k.elguemmat@gmail.com, taleamohamed@yahoo.fr \\ 2Department of Mathematics, Hassan II University, Casablanca, Morocco; h.benlahmer@gmail.com
}

\begin{abstract}
Objectives: To analyze the performance and to determine the most suitable routing type, to ensure the best efficiency in the VANET. Methods/Statistical analysis: Vehicular Ad Hoc Networks (VANET) constitute one of the most promising areas of application of ad hoc wireless networks, able to organize without predefined infrastructure. These networks allow vehicles to communicate with each other or with the roadside infrastructure and will ultimately have safer and more efficient roads through the exchange of timely information to drivers and authorities. Findings: The routing information in VANETs is a major challenge because they are characterized by high mobility resulting in a highly dynamic topology. In this article, we present the most popular routing protocols, offered to do the routing. We describe their main features and functions that ensure the flow of data between different mobile units. We are particularly interested in the problem of delay and bandwidth consumption in routing protocols. In this axis, we compare the various recent proposals for routing protocols to determine the most efficient routing types. This article gives readers a deeper insight on the methods proposed in this area and the most effective solutions to improve VANET. Applications/Improvements: The results observed from this paper motivate to improve the stability of cluster structure in clustering routing protocols in VANETs.
\end{abstract}

Keywords: Intelligent Transport System, Mobility, Routing Protocols, Topology, Vehicular Ad Hoc Networks

\section{Introduction}

In recent years, the research on the Intelligent Transportation Systems (ITS) has been progressing intensively. ITSs are the applications of the new information communication technologies in the transport domain 1 . Among the ITS applications, we can apply it to optimize the use of transport infrastructure and to improve the road safety. In addition, it has the needed information for alerting the driver in case of accidents, congestion, work, emergency braking, parking spaces available, the presence of police radar and emergency response vehicles.
VANETs are simply an application of Mobile Ad Hoc Networks (MANET). They constitute the core ITS whose main objective is the improvement of road safety by taking advantage of the emergence of communication technology and the lower cost of wireless devices. Indeed, by means of installing within vehicle sensors 2 , or at the edge of roads and control centers, vehicular communications will enable drivers to be informed early enough of possible dangers. In addition, these networks will settle more than just improve road safety; they also will offer new services to road users making it a more pleasant road. Interesting contributions on the exchange of information between vehicles have recently been proposed in several

${ }^{*}$ Author for correspondence 
research projects related to road safety. For the establishment of such a network, certain electronic equipment should be installed in vehicles such as environmental perception devices (radars, cameras), a GPS tracking system, and of course a processing platform.

The characteristics of VANET are generally similar to the MANET ${ }^{3}$ technology in the sense that self-organization $^{4}$, self-management, and low bandwidth. VANET is characterized by high mobility of nodes making the highly dynamic network topology ${ }^{5}$. However, in VANETs, nodes tend to move in an organized pattern; thus, portability issues like power consumption and antennas are not significant problems. In general, vehicular network system components consist of an On-Board Equipment (OBE) which is a network device located in the moving vehicle and connected to both wireless network and to in-vehicle network, Road-Side Equipment (RSE), which can be described as a device installed in the side-road infrastructure that connects the moving vehicle to the access network, which in turn is connected to the core network. Vehicular networks have two main types of communications ${ }^{6}$.

\subsection{Vehicle-To-Vehicle (V2v) Communication}

The data collected from the sensors fixed in nodes will be useful in alerting other vehicles about accidents or other emergencies and will also assist the police in tracing criminals $\mathrm{s}^{\mathrm{z}}$. V2V communications are very effective for the transfer of information regarding services related to road safety, but they do not guarantee a permanent connectivity between vehicles ${ }^{8}$.

\subsection{Vehicle to Infrastructure (V2I) Communication}

VANETs can use permanent cellular gateways and wireless access points at traffic junctions for Internet access, gathering traffic information, or routing. In these conditions, the network architecture will be either cellular or WLAN. V2I communication modes are inadequate for applications related to road safety because the infrastructure networks are not performing as to delivery times?

\subsection{The Combination of these Two Modes of Communication Provides an Interesting Hybrid Communication}

Indeed, the increased infrastructure is limited, the use of vehicles as a relay used to extend this distance. Economic purposes and avoiding duplicative terminal every street corner, the use of intermediate hops per vehicle becomes important ${ }^{10}$.

Researchers have proposed several routing protocols for VANET. These protocols are designed to maximize throughput, minimize the delay and packet loss. These routing protocols are divided into six categories [Figure 1]:

- The topology-based routing protocols

- The position-based routing protocols $s^{11,12}$

- Cluster-based routing protocols $\frac{13,14}{14}$

- The Geocast-based routing protocols

- The broadcast-based routing protocols

- Mobicast-based routing protocols.

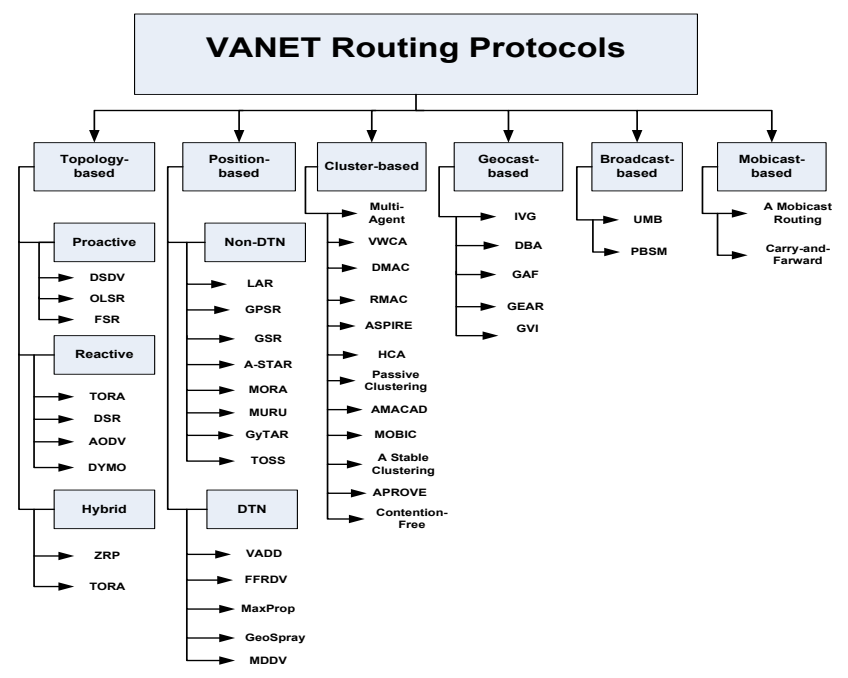

Figure 1. Taxonomy of routing protocols in VANET.

Routing protocols are used in ad hoc communications. They are used to determine the sequence of nodes that the packet must cross to an exchange of information between remote entities. The problems to be met by routing protocols are intermittent connectivity makes them obsolete routes established already, network partitioning that prevents the propagation of packets, delay, and band width consumption.

The VANET networks are based on protocols that provide routing information exchange and communication between vehicles, since it is obvious that improved communication between the vehicle returns to the determination of the effectiveness of these protocols. Therefore, several overviews and surveys have been written, in order to present the VANET research field ${ }^{14-19}$. However, in this paper, we determined the most appropriate type of routing to ensure the highest efficiency of this network. 
Firstly, we studied the different types of routing protocols, performance, and limits VANET to determine the best type of routing for VANET networks. We will present taxonomy, shown in Figure 1 which classifies routing protocols according to their types of routing. Finally, numbers of metrics are selected to compare and analyze of all the existing routing protocols in the literature.

This paper is organized as follows: Section 2 presents the topology-based routing protocols. Section 3 introduces the broadcast-based routing protocols. In Section 4, we detail geo cast-based routing protocols. In section 5 , we review mobicast-based routing protocols. The position-based routing protocols are presented in section 6. Section 7 describes the cluster-based routing protocols. Whereas our discussion is introduced in Section 8, Section 9 concludes this paper.

\section{The Topology-Based Routing Protocols}

The topology-based routing protocol using links in the network to perform packet forwarding. They are classified into three categories: reactive, proactive and hybrid protocols.

\subsection{Proactive Protocols}

Proactive protocols establish and maintain roads for all nodes in the network according to the periodic exchange of routing data. These protocols have the advantage of the availability of routing paths to all nodes. Thus, the packet forwarding delay is very short. However, a traffic control is necessary to update the roads and converge to a consistent state in a network with a dynamic topology. The disadvantage of proactive protocols lies in the cost of maintaining topology information and routing even in the absence of data traffic which implies a continual consumption of bandwidth.

In VANET, nodes have high mobility. The proactive routing protocol is inadequate for this. This routing protocol type may fail in VANET because it consumes bandwidth consumption and can also increase the number of collisions. The strategies realized in proactive protocols are distance-vector routings such as DSDV ${ }^{21}$, link-state routings such as $\mathrm{OLSR}^{22}$ and FSR 23 .

\subsubsection{Dynamic Destination-Sequenced Distance- Vector (DSDV)}

Routing algorithm from Perkins called DSDV $\underline{24}$ was designed specifically for mobile networks. It is based on the classical idea of the Distributed Bellman-Ford algorithm $(\mathrm{DBF})^{25}$. Each node maintains a routing table containing all destinations it can reach and the number of nodes required to reach the destination, and a sequence number associated with the destination node avoiding loop formation. This table is formed by the integration of update data transmitted by each node, which takes place in function of time or according to events related change in the network topology and is either incrementally or full depending on the size of the observed changes. In DSDV, a mobile node must expect until it receives such the next update by the destination to update their distance table ${ }^{21}$. Therefore, the reaction DSDV to changes in the topology is considered slow. Furthermore, this protocol causes significant load control in the network because of update packets sent periodically or in response to events.

\subsubsection{Optimized Link State Routing (OLSR)}

OLSR ${ }^{26}$ applied to VANET networks in the specific environment of a city. Service quality metrics such as delay, jitter, and the loss rate will be analyzed, taking into account the density of traffic, the number of lanes, speed limits and the type of related data. OLSR is a proactive routing protocol optimizing the LSR protocol broadcasts by reducing the notion of Multi-Point Relay(MPR). It aims to provide shortest routes to a destination using the Dijksratechnical. Its innovation lies in how to save radio resources during broadcasts, this is achieved through the use of MPR technique. Therefore, the principle is that each node builds a subset called MPR among its neighbors, which allows achieving all its neighbors in two hops, nodes of this set are used to route and forward messages they receive. The neighbor of a node that is not MPRs, read and process packets but does not broadcast. Only MPR nodes have knowledge of the network topology. Overall, this protocol actually improves LSR avoiding total network flooding but leaves many of its outstanding issues.

\subsubsection{Fisheye State Routing (FSR)}

The FSR protocol ${ }^{23}$ is a link-state protocol that uses the technical fisheye to reduce the number of control messages. In FSR, the fisheye approach translates to maintaining accurate distance and path quality information about the immediate neighborhood of a node, with less detail as the distance increases. In routing, the fisheye approach translates to maintaining accurate distance and path quality information about the immediate neighborhood of a node, with progressively less detail as the 
distance increases. The decrease in accuracy is ensured by changing the update frequencies using different exchange periods for inputs of the routing table. For the entries that correspond to the closest nodes, the exchange period is relatively small. It increases gradually as the nodes become increasingly distant.

\section{Reactive Protocols}

Reactive routing protocols are also called on on-demand protocols because they periodically renew the routing table. They create and maintain routes according to the communications needs of the network. The advantage reactive protocols are that they offer greater adaptability to the topological changes of highly mobile ad hoc network such as VANET. However, these types use a flooding method for route discovery that initiates more overhead and suffers from the initial route discovery method. Thus, they become inadequate for security applications in VANET. Different types of reactive routing protocols are $\mathrm{TORA}^{27}, \mathrm{AODV}^{28}, \mathrm{PROAODV}^{29}, \mathrm{DSR}^{\underline{30}}, \mathrm{BSR}^{\underline{31}}$, and $\mathrm{DYMO}^{32}$.

\subsection{Temporally Ordered Routing Algorithm (TORA)}

TORA ${ }^{33}$ was designed primarily to minimize the effect of topology changes that are common in ad hoc networks. The algorithm adapts to the mobility of these environments by storing multiple paths to the same destination, making topology changes will have no effect on data routing unless all paths to the destination are lost. For this building a road from one node to another will form a directed graph, modeling the different paths from source to destination, and if a node detects a link failure, it will initiate a process that goes Inverting the orientation of the graph, thus preventing the source from borrowing that path. The main characteristic of TORA is that control messages are limited to a reduced set of nodes. This set represents the nodes near the place of the occurrence of the topology change. Each source node knows the path to its destination, but in the case of VANET, this strategy will fail because of the high mobility of the nodes that make the path more dynamic and in this case will get more information disruption. For this reason, TORA is not suitable for the networks which characterize by the high mobility of the nodes.

\subsection{Dynamic Source Routing (DSR)}

$\mathrm{DSR}^{34}$ is designed for use in multi-hop wireless ad hoc networks of mobile nodes. In DSR, network is completely self-organizing and self-configuring, without existing network infrastructure.DSR supplies reactive service to ensure delivery of data packets in spite of high mobility of nodes. DSR protocol includes two mechanisms that operate together to enable the maintenance and of source routes:

- Road Discovery: In this mechanism, a source node sends a packet to a destination node so as to establish a route to the destination. Route Discovery is only used once a node tries to send a packet to its destination and will not know a route to the destination.

- Route maintenance: In this mechanism, the source node is capable of detecting the path to the destination, if the network topology has changed, it cannot move towards a destination node because the link fails. When the road maintenance indicates a source route is broken, the source may attempt to use any other way, it also gets to know the destination, or it can invoke route discovery again to find a new path. Road maintenance for this route is used only when the source is actually sending packets to the destination.

Among the advantages of the DSR protocol in that the transit nodes do not need to maintain updated information to send the data packets because they contain all the routing decisions. In addition, there is a total absence of routing loop because the source-destination is one of the sent data packets. The BSR protocol has improved the DSR performance in case of high mobility. The BSR advantage has reduced the frequency of invoking the discovery phase that is recognized as an important source of traffic control in the protocols on demand. A new routing metric called reliability is introduced to select the backup path.

\subsection{Ad hoc On-demand Distance Vector (AODV)}

$\mathrm{AODV}^{\underline{36}}$ uses the DSR protocol by changing it to avoid having to carry the road ahead in all packets transmitted. Therefore, each node maintains a routing table that specifies for each request source, destination, and the previous node and which is in the RREQ request and is used by reading reversed when returning by the RREP response message. As DSR, the routing tables can be used as caches 
so that an intermediate node knows that the destination corresponds to the source directly. AODV also uses sequence numbers to compare the most recent paths if necessary updates and avoid loops. The use of a timeout to purge the entries of the routing tables to force an update of paths is judged invalid with mobility. Furthermore, Hello messages are sent periodically to ensure the validity of links to emit an RERR error message if no answer to this message indicating the failure of a link. AODV does not ensure the best use of the existing path between the source and the destination. However, recent performance evaluations have shown that there are no great differences between the paths used by the AODV protocol and those used by protocols based on search algorithms shortest path ${ }^{37}$. In addition, AODV protocol presents no routing loop ${ }^{38}$ and avoids the problem counting to infinity of Bellman-Ford, which provides fast convergence when the topology of the ad hoc exchanges network ${ }^{39}$.

\subsection{Dynamic MANET On Demand (DYMO)}

$\mathrm{DYMO}^{32}$ is a new reactive protocol. It is based on routing protocol AODV. DYMO aims to reduce the system requirements of nodes and to simplify the protocol implementation. In addition, route information concerning a requested target, a node will also receive information about all intermediate nodes of a freshly discovered path. There is a major difference between DYMO and AODV. AODV generates route tables for the destination node and the next hop, whereas DYMO keeps route for each intermediate hop. DYMO protocol tried to benefit from the best of previous generation protocols, but with insignificant changes in the approach to the routing problem in ad hoc networks.

\section{Hybrid Routing Protocols}

Hybrid routing protocols are a combination of reactive and proactive routing protocols to make it a more efficient and scalable routing. A hybrid routing protocol has the advantages of distance vector and link state routing protocols and merges them into a new protocol. Typically, hybrid routing protocols are based on a distance vector protocol but contain many of the characteristic and advantages of link state routing protocols. Disadvantages of this type are flooding useless and rising overhead $\operatorname{costs}^{\frac{12}{2}}$. These routing protocols are specifically designed for networks which the nodes are not highly mobility.
The most known hybrid routing protocols are $\mathrm{ZRP}^{40}$ and HARP ${ }^{41}$.

\subsection{Zone Routing Protocol (ZRP)}

$\mathrm{ZRP}^{42}$ is based on the concept of zones. It combines the advantages of both into a hybrid protocol, using the advantage of proactive discovery in the local neighborhood of a node, and employing a reactive scheme for communication between these neighborhoods. ZRP is not a specific protocol as it supplies a framework for other schemes. The separation of nodes local neighborhood with respect to the overall topology of the entire network can apply different approaches and thus take advantage of the characteristics of each technique for a given situation. These local neighborhoods are named zones. Each node can be within several overlapping zones, and each zone may be of a different size. ${ }^{43}$ ZRPconsists of three parts IARP proactive part, IERP reactive moments of it and BRP used with IERP to reduce the query traffic. The source node sends information direct to the destination if they are located in a similar zone, or else the IERP reactive foments path detection.

ZRP provided good results in terms of updates broadcast. It is a combination of proactive and reactive approaches; thereby, it exploited the advantages of them. However, ZRP is not efficient enough in case of high mobility of nodes and urban environment (a lot of obstacles). Therefore, it is unsuitable for VANET.

\subsection{Hybrid Ad hoc Routing Protocol (HARP)}

$\mathrm{HARP}^{44}$ divides the regions into non-overlapping areas and aims to establish a regular route from source to destination to improve the delay. In addition, it implements route discovery between the zones for limited running on the network and selects the best route according to constancy characteristics. In HARP, routing is performed on two levels, namely, intra-zone and inter-zone, depending on the location of the destination. It employs reactive and proactive protocols in inter-zone and intra-zone ${ }^{45}$.

Different from ZRP, HARP concerned with researching and maintaining a path between source and destination, and leaves topology generation to DDR -Distributed Dynamic Routing ${ }^{41,46}$. This separation simplifies the routing protocol and makes the design modular. Different from ZRP, HARP limits flooding to the subset of transfer nodes in each zone. This reduces bandwidth usage and 
the energy consumption of non-forwarding nodes. HARP applies stability at the zone level as route setting parameter that is not the case in ZRP. Unlike previous routing protocol, HARP applies early path maintenance which is more suited for priority classes.

\section{Broadcast-based Routing Protocols}

This protocol uses a simple method to distribute data to multiple nodes simultaneously. Broadcast-based protocol is employed in VANET data sharing, road conditions, weather, and announcements. It works well in a limited number of nodes, but when the number of nodes in the network increases, performance decreases. Each node receives and disseminates the message almost simultaneously, causing collisions, overloading, and band width consumption. Broadcast routing protocol examples are $\mathrm{UMB}^{\underline{47}}$ and $\mathrm{PBSM}^{4 \underline{48}}$.

\subsection{Urban Multi-Hop Broadcast Protocol (UMB)}

$\mathrm{UMB}^{49}$ is a distribution algorithm that modifies the 802.11 access layer in order to be adapted to inter-vehicle communication IVC. This adaptation uses RTS / CTS mechanism coupled to DDT type of mechanism that avoids the problem of hidden nodes broadcasting mode. Unlike flooding streaming protocols, says UMB packet sending and reconnaissance operations to the more distant do not allow a priori knowledge of network topology. UMB is divided into two stages, the first called directional broadcast where the source node selects a node in the diffusion direction for a data relay. The second phase, the local diffusion at the intersections to disseminate the packets in all directions, because UMB uses transponders in the intersections to forward packets to all segments. The main advantage of UMB protocol is the multi-hop broadcast reliability in urban canals $s^{50,47}$. However, it required for each intersection a fixed receiver installation. In addition, this installation cost high in term of time and materials in case of the extended network.

\subsection{Parameterless Broadcast in Static to Highly Mobile (PBSM)}

$\mathrm{PBSM}^{51}$ is an adaptive broadcasting protocol that does not require nodes to know about position and movement of their nodes and itself. It uses Connected Dominating Sets (CDS) and neighbor elimination concepts to eliminate redundant broadcasting. It employs two-hop neighbor information obtained by periodic beacons to construct CDS.

$\mathrm{PBSM}^{52}$ uses the information of the 2-hops neighbors obtained by the exchange of the periodic messages to construct CDS. A vehicle X maintains two routing tables: $\mathrm{R}$ and $\mathrm{NR}, \mathrm{R}$ containing the neighbors that have already received the broadcast packet and NR the others. After the expiration of a timer, the vehicles $\mathrm{X}$ broadcasting the packet if the table NR is not empty. ACKPBSM ${ }^{\underline{53}}$ was proposed to minimize the control packet overhead in data forwarding.

PBSM and ACKPBSM use to store and forward method to deliver the packet to the network that uses high delay which is not adequate in safety application for VANET ${ }^{51}$.

\section{Geocast-based Routing Protocols}

Geocast-based routing protocols ${ }^{54}$ keep the principle of routing data packets from a single source vehicle to all vehicles belonging to the destination zone knew zone of relevance ZOR. However, to replace the simple flooding of the geocast message from the source to ZOR, a forwarding zone knew zone of forwarding $\mathrm{ZOF}$ is used to confine the message forwarding until it reaches ZOR ${ }^{55}$. This protocol is failed in the case where no vehicle is in the geographical zone. The various Geocast routing protocols in VANET are IVG ${ }^{\frac{56}{6}}$, DRA ${ }^{\frac{57}{7}}$ and GVI ${ }^{58}$.

\subsection{Inter-Vehicle Geocast (IVG)}

$\mathrm{IVG}^{56}$ is a new method of distribution that generalizes methods TRADE and DDT. It overcomes the network fragmentation problems, reliability, and calculating neighbors. IVG ${ }^{59}$ is a protocol designed to solve problems such as determining the direction, location in space and dissemination of information, which are due to the high mobility of nodes. It is based on sending an alarm message to neighbors during an accident even the vehicles located in the extremities of the transmission range send the message. The purpose of this protocol is to avoid collisions so that the loss of information and network overload. IVG protocol is based on the calculation of deferring Time and Teta that is calculated according to the distance between nodes including the antenna transmission range so as to minimize the number of broadcast message. 


\section{Dynamic Backbone Assisted (DBA)}

$\mathrm{In}^{60}$, propose a Dynamic Backbone-Assisted (DBA) MAC protocol as a solution to help geocast communication on highway environments for different categories of vehicular applications. DBA-MAC combines elements of classical proactive and reactive schemes through a cross-layer architecture which includes: (i) a novel distributed clustering algorithm and (ii) a fast information dissemination mechanism. First, DBA-MAC creates and maintains a virtual backbone of vehicles inside the highway scenario. After that, it provides fast dissemination of geocast messages through a combination of contention-free and contention-based forwarding mechanisms at the link layer. It has investigated different metrics of backbone creation to support different classes of vehicular applications.

\section{Geographical Adaptive Fidelity (GAF)}

GAF $\underline{61}$, one of the topology management protocols, is applied to manage the states of network nodes and select the multi-hop relay nodes which can improve energy efficiency. Each node is assigned to a given area according to its geographical coordinates. The nodes belonging to the same zone are considered equivalent in routing cost. Thus, only one node of each zone is chosen to route the data, and the others are set to sleep mode to conserve energy. This step will increase the lifetime of the network as a function of the total number of inactive nodes. In GAF, the node can be found in one of three possible cases: (1) Discovered mode, to determine its zone neighbors. (2) Active if involved in data routing, and (3) Asleep if not involved in routing. To support mobility, each node estimates and diffuses the time necessary to leave its zone to its neighbors. Based on this time, they estimate their times to choose relay node. GAF does not take into account the energy of the node when choosing the representative, which can generate holes in the network once the latter depletes its energy ${ }^{62}$.

\section{Geographic and Energy Aware Routing (GEAR)}

GEAR $^{\underline{63}}$ is considered an improved $\mathrm{DD}$ protocol based on geolocation and energy of neighboring nodes. The main idea is to limit the number and to distribute the interest in specific areas rather than the entire network. This way, energy conservation is achieved compared to DD. To choose the best path routing, each node calculates the cost: a cost that is a combination of residual energy and distance to the destination. In addition, another acquired (learning) which consists in an adjustment of the first cost taking into account the routing through the holes that can appear on the network. A hole appears when a node has no neighbor on a path to the destination. The cost is spread acquired in the opposite direction each time a packet is received by the recipient to adjust the cost of the route for packets that follow. Two phases are identified in this algorithm:

1. Data dissemination to the destination area: in this case, the received packet will be routed through the nearest node in the recipient's area. If there is no neighbor node apart from the node itself (if a hole) from a neighboring node will be chosen based on the acquired cost.

2. Data dissemination in the recipient's area: Once the packet arrives in the area concerned it is distributed either in a controlled manner or recursively geographically by sharing in each iteration the area into four zones until obtaining zones with a single node. It should be noted that the controlled release is effective on the less dense networks; while the recursive method is more profitable otherwise.

\section{Geo-localized Virtual Infrastructure Mechanism (GVI)}

GVI ${ }^{\underline{64}}$ consists of selecting vehicles that will perpetuate information broadcasting within the intersection area. Therefore, GVI mechanism is composed of two phases:

1. The first phase is selecting vehicles able to reach the broadcast area. This phase involves selecting vehicles that have just received a packet of data after a local broadcast, and are located in the notification area, and are able to reach the center of the intersection.

2. In the second phase, among nodes, one node will be selected as the local broadcaster. This is the vehicle that has estimated travel time to reach the diffusion region closest to the desired broadcasting period. Therefore, there is guarantee that the chosen vehicle broadcasts the packet; it is close to the center of the 
geocast area. GVI can fail in the particular case where no vehicle is located in the notification area at the last local broadcast. This depends on mainly road traffic conditions and choice of the broadcast period.

\section{Mobicast-based Routing Protocol}

A Mobicast-based routing protocol ${ }^{65}$ is a new spatiotemporal geocast routing protocol for VANET. Contrary to the classical geocast-based protocol, mobicast-based protocol takes into account the time factor. The principal objective of the mobicast protocol is the data dissemination to all vehicles which must be in a prescribed area of space at any given time. It is meant to help applications requiring spatiotemporally assortment in VANET. The feature of a mobicast is to send a mobicast message to nodes situate in a geographic zone at time $t$, where it is denoted as the Zone of Relevance (ZORt). Nodes situated in ZORt at the time $t$ must receive the mobicast message.

\subsection{A Mobicast Routing Protocol}

The authors ${ }^{66}$ propose a new spatiotemporal multicast routing for facilitating applications requiring spatiotemporal coordination in VANETs. The main of this article is improved a mobicast protocol, to estimate dynamically the specific ZOF and to disseminate mobicast messages to all vehicles in ZOR. To overcome the temporal network fragmentation problem, it has developed an algorithm by extending some adaptive to improve the dissemination rate; although the connectivity of ZOR is temporarily lost because any vehicle in ZOR suddenly accelerates or decelerates its speed.

\subsection{A Mobicast Routing Protocol with Carry-and-Forward}

$\mathrm{In}^{\frac{67}{}}$, present a mobicast algorithm for a highway environment. The vehicles are situated in a zone of relevance (ZOR) at time $\mathrm{t}$ on which the mobicast routing should disseminate data initiated from a source vehicle to all vehicles that have never appeared in ZOR at time $t$. This data dissemination should be made by the time $t+\lambda$ of the carry-and-forward technique. Furthermore, the temporary network fragmentation problem is taken into account the protocol design. In addition, the low degree of the channel has retained to reserve the resource for safety applications.

\section{Position-based Routing Protocol}

In position-based routing $\underline{68}$, all vehicles acknowledge their own positions and their neighbor node geographic locations through position pointing devices such as GPS. It does not manage any routing table or exchange information related to the link state with neighboring nodes. The position information is used in making routing decisions. Position-based protocols are divided into two types: non-delay tolerant network (non-DTN) routing protocols and delay tolerant network (DTN) routing protocol.

\subsection{Non-Delay Tolerant Network (Non- DTN)}

The non-DTN protocols do not regard a disconnectivity problem; it assumes there are always a number of nodes to achieve the successful communication. Therefore, this protocol is suitable for a high density of nodes. These protocols are tested at transmitting immediately the data packets to the destination. However, non-DTN approach may be unsuccessful if there is no closest neighbor to the destination rather than the current node itself $\frac{68}{}$. The following is an overview of all non-DTN routing protocols in VANET. Examples of Non-DTN routing algorithm are $\mathrm{LAR}^{\underline{69}}, \mathrm{GPSR}^{\frac{70}{}}, \mathrm{GSR}^{\underline{71}}, \mathrm{~A}^{\mathrm{ST}} \mathrm{SAR}^{7 \underline{72}}, \mathrm{MORA}^{\underline{73}}, \mathrm{MURU}^{\underline{74}}$, $\operatorname{GYTAR}^{\frac{75}{5}}$ and $\operatorname{TOSS}^{\frac{76}{6}}$.

\subsection{Location-Aided Routing (LAR)}

$\mathrm{LAR} \frac{69}{}$ is a reactive protocol, which proceeds in a similar way to DSR protocol by using a road construction mechanism. The main difference between two protocols is that LAR uses the location information in order to avoid flooding of route request packets to a geographical area, calculated according to movements of two protagonists of the exchange.

\subsection{Greedy Perimeter Stateless Routing (GPSR)}

GPSR $^{70}$ uses the position of the nodes and the destination to decide the next node to transmit the packet. A node employs the greedy mode and forwards the packet to 
its closest neighbor of the destination. Knowledge of its neighbors is through periodic broadcast Hello messages containing the node ID and its position.

\subsection{Geographic Source Routing (GSR)}

In GSR ${ }^{71}$, a source node that wishes to transmit a data packet to a target vehicle calculates the shortest routing path to reach this vehicle from a geographic information map. It should be noted that the routing path is calculated in its entirety by the use of Djikstra algorithm. The source vehicle selects a sequence of intersections by which the data packet should pass to reach the target vehicle. This sequence of intersections is formed by a set of fixed geographical points of passage of the data packet. To send the data from an intersection to another, GSR proposes to use a greedy mode.

\subsection{Anchor based Street and Traffic Aware Routing (A-STAR)}

A-STAR ${ }^{72}$ is similar to GSR by adopting a protocol routing that takes into account the anchor of street features. However, unlike the anchor GSR calculates paths depending on the traffic. A weight is assigned to each road in function to its quality that is a small or large street that is serviced by a number of buses. Roads of information provided by the bus give an idea of the traffic load in each street which gives an idea of the city at different times. It uses the main streets that the Connectivity on these streets may be higher due to the high density of nodes and more stable due to the presence of regular city bus. When choosing a road with a high density without regard to its width leads to delays in the case of a large-scale road.

\subsection{Movement-Based Routing Algorithm (MORA)}

MORA $^{73}$ exploits position and direction of movement of vehicles to adjust retransmission decisions to the context of vehicles, and thus to face the high mobility of node and the relatively frequent changes in the topology. This approach has developed a function which depends on the transmission distance between the source, the destination and the moving direction of the node. The source node see a package including a route request to the destination node to the source node responds with a road package including information moving.

\subsection{Multi-hop Routing Protocol (MURU)}

MURU $^{74}$ is a reactive routing protocol based on movement and adapted to urban environments. It uses a metric called level disconnection expected to assess the quality of the path. This metric is calculated using the information on the prediction of the speed and the trajectory of each vehicle. MURU is optimized with a power reduction mechanism to reduce the overhead of bandwidth by removing unnecessary control messages. However, when the density of nodes increases the end-to-end delay decreases.

\subsection{Greedy Traffic-Aware Routing Protocol (GyTAR)}

$\mathrm{GyTAR}^{25}$ is a junction-based routing protocol that is able of finding robust routes that are designed to function optimally within urban environments. It integrates the IFTIS mechanism for routing packets. GyTAR is able of selecting robust routes with high connectivity in order to inject data packets. It uses improved greedy approach to relay the data packets between two successive intersections; this approach makes it possible to minimize the number of hops to reach a destination intersection.

In GyTAR, the greedy routing strategy is used to send the packet between two implicated intersections. For this, using GyTAR makes a packet move closer sequentially to the destination along roads where a suitable number of vehicles offer connectivity ${ }^{77}$.

\subsection{Token-Passing based Multi-Point Relays (TOSS)}

TOSS $^{76}$ is a node discovery process more reliable relays which are responsible for disseminating messages to the destination node. This protocol provided better delivery reliability successfully messages to neighboring nodes. However, when the number of neighbor nodes increases, the complexity increases because each mobile entity moves independently with a variable speed, and the packet size increases.

\section{Delay Tolerant Network (DTN)}

The DTN architecture model has been applied for vehicular Networks called Vehicular Delay Tolerant Networks (VDTN). In these networks, vehicles use a message relaying service by their mobility in the network and collect 
messages from source nodes. The known of these protocols are VADD ${ }^{78}$, FFRDV $\stackrel{79}{ }$, MaxProp ${ }^{80}$, GeoSpray ${ }^{81}$, and MDDV 50 .

\subsection{Vehicle-Assisted Data Delivery (VADD)}

$\mathrm{VADD}^{78}$ is routing protocol in delay tolerant networks. This protocol aims to improve routing by using the carry-andforward technique based on the predictable vehicle mobility. At each intersection, the vehicle will decide and will select the next forwarding path with the minimize delay. This delay depends on the road distance, average speed, and density of nodes. To solve the problem of low connectivity between nodes, a vehicle in motion carries the package until it reaches a new vehicle is moving in its neighborhood and transmits the package. However, this relay strategy can fail in the case where no neighbor vehicle carrier vehicle. Thus, this protocol does not function in a low density of nodes.

\subsection{Fastest-Ferry Routing in DTN-enabled VANET (FFRDV)}

FFRDV $\underline{79}$ utilizes the concept of sending applications data which can tolerate delay, over the fastest ferry. It considered that the road is divided into logical blocks of some fixed size. While entering the block, the ferries which have the data broadcast a Hello message. All ferries within the block reply to this message with their speed and coordinates. The ferry has data, called current ferry (CF). After receiving Hello messages from all ferries, CF compares it speed from all speed in reply Hello message. Indeed, CF selects the fastest ferry among all ferries within the block. Fastest ferry is called designated ferry (DF). The CF sends Bundle to DF and discards Bundle after receiving acknowledgment from DF. The CF after discarding Bundle becomes normal ferry ${ }^{82}$.

The velocities of vehicles are compared within one block. At the initialization of the network communication, every vehicle creates one state-report, which includes the current position and velocity. And the state report is updated periodically. The node is called message ferry only when it's carrying data. Once the bundles are forwarded and acknowledged, the ferry will discard the data, and change to be a normal mobile node. Ina block, the priority of vehicle selection is decided by maximum velocity $\underline{\underline{82}}$. This protocol improved packet delivery. However, FFRDV does not determine their efficiency in end-to-end delay and bandwidth.

\subsection{MaxProp}

MaxProp ${ }^{80}$ protocol is based on a store-carry-forward mechanism which is usually utilized in a DTN environment. However, it proposed an approach which enables the nodes to assign the priority of packets. On the basis of the given priorities, each node can decide either to transmit or to drop the packet. In vehicular DTNs, the transmission duration and opportunities for each node are limited, since the nodes move fast in sparse areas. Furthermore, the buffer of node is also limited in a real environment. Therefore, to decide the priority of packets in a buffer of nodes is important when performing efficient routing. MaxProp introduced a mechanism to handle old data within the network. In MaxProp, each packet stores a hop list of nodes that the packet already traversed. This hop list enables each node to identify the age of packets. The packets that characterized by lower hop list values, they are considered to be new packets and thus higher priorities are assigned to them. In the case of a node encounter, the packets that characterized by highest priority, they are transmitted, and the remaining packets are transmitted later. On the other hand, the packets which have the lowest priority will be deleted first in case a buffer is full $\frac{83}{}$.

\subsection{GeoSpray}

GeoSpray ${ }^{81}$ takes routing decisions based on geographical location data. It also combines a hybrid approach between multiple-copy and single copy routing schemes. GeoSpray performs control spraying by distributing a limited number of bundle copies to the network nodes that approaching the destination. Afterward, it passes to forwarding scheme where it combines several control data sources to effect routing decisions. Finally, in order to improve resource utilization, it clears delivered bundles across the network nodes. GeoSpray improved resource utilization. Furthermore, it clears delivered bundles across the network nodes.

\subsection{Mobility-Centric Data Dissemination Algorithm for Vehicular Networks (MDDV)}

$\mathrm{MDDV}^{50}$ is designed to exploit the vehicle mobility to transport data from source to destination. It combines the idea of opportunistic forwarding, transfers according to trajectory and geographical transmission. 
Opportunistic forwarding is used when the network is fragmented. In general, MDDV has two phases: the transfer phase and the propagation phase. In this protocol, when no vehicle can be found by along the forwarding trajectory, a carry-and-forward scheme is employed. The vehicle which is incapable of transmitting the data packet will store it until it finds a more appropriate relay. MDDV is well suited for delay tolerant applications where users can tolerate some level of delay, as long as the data eventually arrives. This protocol effectively reduces redundancy, but without improvement for the probability that a collision occurs.

\section{Clustering-based Routing Protocols}

The clustering-based routing protocol consists of organizing the nodes of the network into virtual groups called clusters. The communication is divided into cluster member to cluster-head and cluster-head to cluster-head communications. The nodes, geographically neighboring, are grouped in the same cluster according to certain rules. In a cluster, there are usually three types of nodes: cluster-head $(\mathrm{CH})$, gateways, and cluster member $(\mathrm{CM})$. In each cluster, a node is selected or elected as a $\mathrm{CH}$, which has additional functions (routing, access to the medium, etc. $)^{84}$. Clustering routing protocol examples are APROVE $E^{\frac{85}{}}, \mathrm{VWCA}^{\frac{86}{}}, \mathrm{DMAC}^{\frac{87}{4}}, \mathrm{RMAC}^{\frac{88}{}}, \mathrm{ASPIRE}^{\frac{89}{}}$, $\mathrm{HCA}^{90}$ and MOBIC ${ }^{91}$.

\subsection{A Multi-Agent Approach}

The authors propose a multi-agent approach for routing in vehicular networks. The new system is based on four agents to find the best path, to send data packets, and to reduce traffic. Each agent executes its role in collaboration with other agents. The officer examines collects context information and communication environment to calculate the route. This agent named context-agent. Moreover, in different routes available to convey a packet, it uses an agent to determine various aspects of these routing systems to choose and to compare the most suitable in order to select a better cluster. The agent calls the agent optimization, it's the primary objective of this agent is to link the poles represented by an agent named cluster-head agent and to improve communication between them depending on the context information collected $\underline{22}$.

\subsection{Vehicular Clustering based on the Weighted Clustering Algorithm (VWCA)}

$\mathrm{VWCA}^{\frac{86}{}}$ is a clustering algorithm that employs a complex metric to improve connectivity and stability of the cluster. This metric is calculated from the value of distrust the number of neighbors on the basis of the dynamic transmission range and vehicle travel direction. Using the value of distrust vehicles have a lower value of distrusting their neighbors, are elected as cluster-head. Therefore, the cluster-head are safer vehicles that other network vehicles. VWCA presents an algorithm that monitors the behavior of vehicles on the network to discover the abnormal vehicle.

\subsection{Distributed and Mobility-Adaptive Clustering algorithm (DMAC)}

$\mathrm{DMAC}^{87}$ is an algorithm that can adapt to changes in the network topology and mobile environments. The main idea of the algorithm is that the node with the highest weight in its neighborhood is chosen to be a clusterhead. Each node is assigned a generic weight which may depend on a set of knot parameters. The objective of the algorithm DMAC is to improve the stability of the clustering avoids reclassification when clusters of vehicles are moving in different directions. Each node needs to know its direction, current location, and speed, through by GPS or a similar service. The freshness metric is the safety factor for unnecessary reclassifications. This factor is calculated between two vehicles to receive their data movement direction and Hello messages. It indicates the expected time nodes, which will be capable of communicating and prevents reclassification in the cases where the direction is the same, although the communications time to be short, in the case of on overtaking. The lifetime of messages also has the construction of multi-hop clusters, which is a rare characteristic ${ }^{93}$.

\subsection{Robust Mobility Adaptive Clustering (RMAC)}

RMAC $^{88}{ }^{i}$ s specifically designed for VANET networks where nodes move at high speed. RMAC uses the speed, location, and direction to calculate the relative mobility value for elected a cluster-head. It was designed to meet the following requirements: The system must be able to meet the smallest to the largest networks, the algorithm 
must operate under conditions, where node mobility is high or average with higher speeds $30 \mathrm{~m} / \mathrm{s}$. RMAC uses cluster-heads to synchronize the exchange of control packets. The algorithm could work in an environment without a fixed infrastructure. Though, nodes can assume different roles within clusters over time. Ultimately, after a communication backbone is established, the system must be capable of supporting multiple geographic routing hops by maintaining and providing accurate information on the 1-hop neighbor.

\subsection{Adaptive Service Provider InfrastructuRE (ASPIRE)}

ASPIRE $^{89}$ adopts the idea of serving the personal mobile nodes by other mobile nodes, which reduces costs and increases the efficiency of network throughput, and is used in the design based on Wi-Fi. However, ASPIRE solves the problems of transfer and overhead and limits the length of the path between any source-destination pairs. Its goal is to provide high connectivity and to create large clusters

\subsection{Hierarchical Clustering Algorithm (HCA)}

$\mathrm{HCA}^{90}$ is another clustering algorithm. It reduces the number of collisions by simultaneous transmissions in the same cluster, transmissions are only allowed on slots assigned by the cluster-head. The algorithm consists of four phases. The first three phases are referred to a static scenario, while the fourth stage processes the topology changes. The nodes are not required to move in a particular mobility scheme but then can move freely. Thus, the algorithm can be used in VANETs or any other network that consists of mobile entities. It characterizes by its simplicity because it does not use location services. Therefore, it does not support all improvements related to the shape of the node movement that could optimize the duration and stability of cluster-head.

\subsection{Passive Clustering Based Techniques for Inter-vehicle Communications}

The authors proposed a clustering routing protocol to compare passive clustering techniques with three different steps: vehicle density, the durability of the link, and link quality. The vehicle density is calculated using an effortless approach. Each vehicle periodically transmits advertising packets to its neighbor's entire only one hop to obtain the vehicle density. To measure the quality of the link, it uses the ETX metric, which indicates the quality of bidirectional transmission of a link. The expiration time is used as a link metric durability ${ }^{94}$.

\subsection{Adaptable Mobility-Aware Clustering Algorithm (AMACAD)}

AMACAD ${ }^{95}$ supports the speed, the relative destination, final destination and current location as metrics for calculating the parameter in an urban environment. AMACAD based on the destination to improve stability in VANETs. AMACAD operates in a distributed manner, where each node runs the algorithm. All vehicles employ a GPS or a navigation system (NS). The destination is divided into two types:

1. The relative destination is the nearest destination based on the current region.

2. Final destination is recorded in the GPS or NS.

The vehicle destination is the key factor, where the clustering decision considers this factor. Cluster size is variable depending on vehicle density, speed and required minimum bandwidth or quality of service where parameters can be predefined or provided on the fly from vehicle sensors and application profiles ${ }^{15}$.

\subsection{A Mobility Based Metric for Clustering (MOBIC)}

MOBIC ${ }^{91}$ is a cluster-based protocol designed for MANET, which also operates in VANETs. MOBIC presented a new measure relative mobility of nodes in a MANET based on the relative power levels because of successive packet receptions to a node in the case of the mobility value it uses the most low-ID algorithm. It demonstrates that the relative mobility is a better criterion for classification rather than IDS that are not representative of node mobility in a MANET. However, MOBIC is suitable for stable cluster training in situations involving mobility both low and high.

\subsection{A Stable Clustering Algorithm for Efficiency Applications}

The authors propose a cluster-based algorithm for VANETs that is a communication protocol of a hop based on the 
metric of direction. The algorithm assumes that each vehicle knows its position using GPS and uses digital maps that determine the direction of the vehicle. The objective of this algorithm is to reduce delay in the system without affecting the stability of clusters. First, change the policy for the election of cluster-head. On the other hand, when forming clusters, it introduces the concept of lifetime to check the connection duration between the cluster-head and nodes. Cluster formation is formed before the intersection and is based on the intended travel course ${ }^{96}$.

\subsection{Affinity PROpagation for VEhiclar networks (APROVE)}

APROVE ${ }^{85}$ forms clusters using the Affinity Propagation algorithm in a distributed manner. It presents a new mobility-based clustering scheme for VANET. This algorithm takes into account the mobility and stability. Cluster performance was measured in terms of average $\mathrm{CH}$ duration, average cluster member duration, the average number of clusters, and the average rate of cluster-head change. Each node transmits messages of responsibility and availability to its neighbors and then takes a decision on the clustering independently. For example, each node i periodically broadcasts a Hello message containing all the necessary information to APROVE algorithm. The Hello packet for the node i contain ID, position, speed, cluster status, availability, and responsibility for each neighboring nodes i and the current state of convergence cluster. A node i calculates responsibility for each neighbor $j$ and its availability for each neighbor $j$. APROVE present two different procedures for selecting and maintaining the cluster-head. It uses a synchronous time interval CI where all nodes have their cluster-head of decision in each CI. The second APROVE asynchronous method does not require time interval for cluster-head decision. Simulation presented a minimization of the relative mobility and the distance of each cluster-head to members of the cluster. The clusters created are stable and have a long average duration of a cluster member, a long average cluster-head, the low average rate of cluster-head variation, and reasonable overhead. However, when the network density increases the size of the neighbor list becomes sufficiently large enough, APROVE will overload bandwidth.

\subsection{Novel Cluster-based Contention-Free Broadcast Protocol}

The authors proposed a new reservation protocol time intervals, where cluster-heads are centralized and adapt continuously to the vehicle dynamics. Thus, the uses of such centralized authors protocol ensure efficient use of time slots for the exact number of active vehicles, including hidden vehicles. This protocol also provides for limited delay security applications to access the communication channel, and it reduces the overhead using a directed broadcast propagation and one reservation request for periodic access to the medium is used during the period of stability during which nodes remain in the same cluster and cluster-head does not change its state ${ }^{97}$.

\section{Discussion}

Routing in VANET is a very difficult problem that poses challenges for many researchers. To ensure that vehicles can communicate, it must define the routing protocol. The objectives of VANET are to achieve an efficient routing protocol that satisfies the following criteria: minimize the end-to-end delay, minimize the consumption of bandwidth, and avoid collisions in the conditions of the high mobility of nodes and changes topologies. Several studies have focused on the creation of a protocol that verifies these goals and adapted a dense environment, mobile and large scale. In this article we presented a survey of routing protocols in VANET, we present the problems of routing in vehicle networks and describe the different solutions for routing and illustrate the problems associated with these protocols. We made a comparison between its different types of routing as shown in Table 1.

In AODV routing protocol, if a source node seeks its destination, it sends an RREQ to a limited number of hop. If the source node does not receive a response after a specified timeout, it retransmits another search message by increasing the maximum number of hop. This procedure is repeated a maximum number of times before declaring that the destination is unreachable. Therefore, AODV uses flooding to discover the roads, which can generate a huge traffic control. The problem is that the systematic replay messages for discovering the roads unnecessarily because excessive consumption of bandwidth since each node will receive the same information several times over the wireless channel. Unfortunately, AODV is very simple approach and is not efficient because it requires the participation of all nodes, whereas this is not always necessary. Therefore, it leads to a lot of redundant messages, which will have several consequences, broadcast storms, hidden nodes, lower reliability, and higher consumption of end-to-end delay. 
Table 1. Comparison of VANET routing protocols

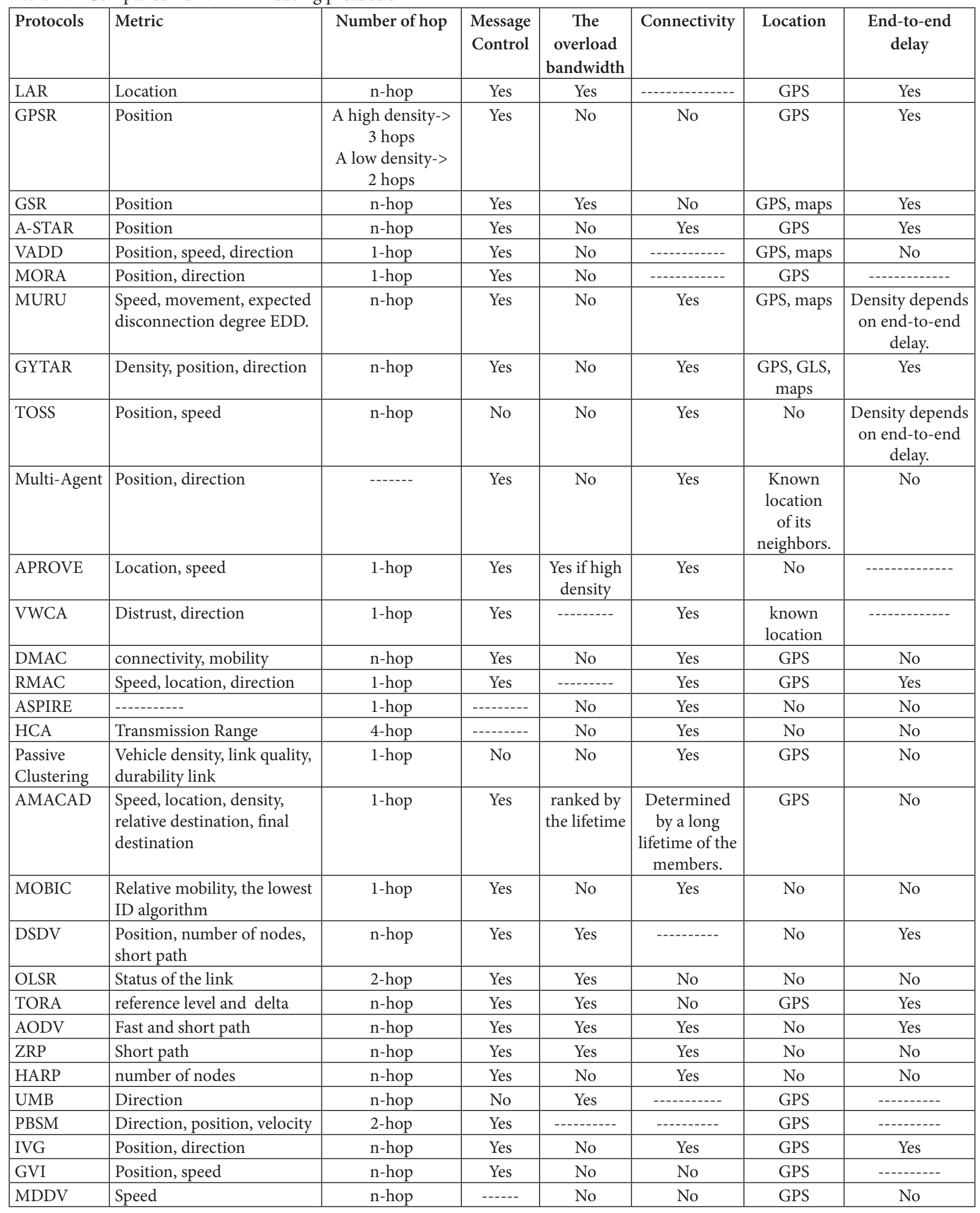


The authors performed a comparison of performance between the protocols AODV, DSR, FSR, and TORA. After analyzing the TCP throughput, the rate of delivery and the average delivery of packets, it is shown that TORA is not suitable for vehicular environments, whereas AODV and FSR have shown encouraging results only in the case of urban scenarios. The results also showed that in the case of low traffic density, the throughput of FSR is less than AODV and DSR. The two protocols do not charge to the relative network, but especially a problem because DSR further end-to-end delay ${ }^{98}$.

$\mathrm{In}^{99}$, an extensive study that compares the routing protocols AODV, DSDV and DSR in highway scenarios were made. These results have demonstrated once again that these routing algorithms are not appropriate in the VANET scenarios from the perspective of rate of delivery, routing, and load end-to-end delay.

In a proactive routing protocol, the network topology is known to all mobiles. The routes are available immediately; on the other hand, we must regularly disseminate information on the network topology changes ${ }^{38}$. Unfortunately, the replay useless information leads to a lot of redundant messages, which will have several effects, namely collision problems, hidden nodes, higher consumption of bandwidth, slower convergence, and consumer end-to-end delay. Proactive protocols have permanently a route for every destination in the network, but in return generate a significant volume of traffic ${ }^{38}$.

In a reactive routing protocol, mobile nodes retain substantially no information about the overall network topology. Only stores information about the active routes. Roads are built to order and are destroyed when no longer used ${ }^{38}$. Among the advantages of reactive routing protocol, does not consume the bandwidth and generates a low traffic volume but in return generate a delay in the reconstruction of roads and more difficult to produce optimal routes.

Hybrid protocols ${ }^{100}$ is suited to large-scale networks. Nevertheless, this type combines the disadvantages of the proactive and reactive protocols, such as regular flooding and the exchange of control packets to search for a route to a remote node.

The broadcast routing protocols have a very simple algorithm for broadcast packets, but they become useless in a very dense environment. These protocols are also less efficient in terms of bandwidth since the nodes broadcast packets to all destinations, consumers more end-to-end delay, collision probability and the problem of hidden nodes.
A-STAR collects information on traffic density provided by bus without considering delay and the flow of traffic, but bus travel are limited. By contrast, VADD used against paths with a lower delay, but it did not give how can calculate the delay estimate. A-STAR and VADD do not take into account the density of vehicles in real time. The solution is comprised using those posting messages to calculate the delay between two successive packets sent and to eliminate non-useful messages to gain in terms of overload bandwidth.

GPSR has improved LAR in which it uses the relay nodes to reach the final destination if the traffic density increases, in this case, the number of hops increases consequently will further delay. The second disadvantage when choosing a destination closer to the node, it does not take into account the speed and direction of movement of vehicles when the dynamics increase the information on the recorded position it is essential to ensure the urgent message applications will have a lifetime limited. Therefore, the choice of vehicle is not optimal in the sense that the selected vehicle is not necessarily the vehicle closest to the destination at the time of sending the packet. Then delays increase. Therefore, these protocols must be further improved to reduce the end-to-end delay. We can use a solution which consists in reducing the delay by using the speed as a metric to estimate the position of the nodes.

After the study that was done on the position of routing protocols, broadcast, geocast and topology deduce that this type of routing suffers end-to-end delay, collision, and connectivity between nodes are extremely low in the flow of traffic and problems of knots caches.

There are protocols clustering offers a technique to reduce the probability of collision and packet delivery rate $^{97}$. On the other uses different parameters, such as speed, displacement of the nodes, calculates the value of the entropy ${ }^{\frac{86}{6}}$, density of vehicles and connectivity ${ }^{94}$, the mobility value ${ }^{\frac{88}{2}}$, etc. to measure the stability of the nodes. Clustering algorithms have objectives such as the stability of the clusters, the formation of groups, minimize the end-to-end delay, and provides a good quality service. The main objective of clustering algorithms is to maintain the structure of clusters as stable as possible over time. The stability of the cluster structure ensures the stability of roads and thus reduces the cost of maintenance. Clustering routing protocols have significant benefits in the design of the gateway for routing. In each cluster, a node is elected to act as the gateway to other clusters. This 
algorithm divides a network into several clusters, where the intelligent use of bandwidth $\frac{101}{}$.

After the comparison between the different types of routing deduced that clustering routing protocols improve VANET. It is important to improve the value of stability and putting into account the change in the topology and node mobility during the interval of time to avoid the disruption of packets $\frac{84,102,103}{}$.

\section{Conclusion}

VANETs have a wide study because they introduce new applications such as intelligent transport systems. VANET provides communication services between vehicles or infrastructure located on roadsides. Routing is a basic function essential to the functioning of the networks. The purpose of routing protocols is only to specialize with routing messages based on several criteria. However, routing in VANET has problems of instability of the roads caused by the high mobility of nodes and communication problem in environments where there are obstacles and empty is the case in an urban environment. In this article, we studied the proposed routing protocols for VANET. We discuss the different solutions for routing and illustrate the problems associated with these protocols. Table 1 summarizes the characteristics of the routing protocols (that is to say metric, the number of hops, the overloading of bandwidth, connectivity, and how to determine the position information). The positions and clustering protocols are used with respect to other routing protocols in VANET. Position-based protocols cannot respond to the dynamic network topology, which is considered a challenge. It was found that clustering protocols are effective to send a packet within a short time and without collisions.

Many studies have focused on the development of clustering protocols to support an efficient and reliable communication between vehicles. However, we found that several important challenges have not been solved and require extensive research. For example, in terms of the stability of cluster-heads, so we must concentrate to seek a factor that calculates the stability of nodes.

We believe that our investigation will be useful to the research community and will serve as an introduction to a document suitable for people who want to continue the study and application of VANET. Research is concentrated on clustering solutions that exploit some measure of vehicle traffic. We are working on identification of the parameters to form more stable clusters; therefore, the connectivity of the vehicle can be further improved.

\section{References}

1. Barrero F, Guevara JA, Vargas E et al. Networked transducers in intelligent transportation systems based on the IEEE 1451 standard. Computer Standards and Interfaces. 2014; 36(2):300-11.

2. Raya M, Hubaux J-P. The security of vehicular ad hoc networks. In the Proceedings of the 3rd Association for Computing Machinery (ACM) Workshop on Security of Ad Hoc and Sensor Networks, Alexandria, VA, USA; 2005 Nov 7. p. 11-21.

3. Singh S, Agrawal S. VANET routing protocols: Issues and challenges. In the Proceedings of the Recent Advances in Engineering and Computational Sciences (RAECS), Institute of Electrical and Electronics Engineers (IEEE); 2014 Mar 6-8. p. 1-5.

4. Ayyash M, Alsbou Y, Anan M. Introduction to Mobile Ad-Hoc and Vehicular Networks. In the Proceedings of the Wireless Sensor and Mobile Ad-Hoc Networks. Springer, New York; 2015. p. 33-46.

5. Namboodiri V, Agarwal M, Gao L. A study on the feasibility of mobile gateways for vehicular ad-hoc networks. In the Proceedings of the 1st Association for Computing Machinery (ACM) International Workshop on Vehicular Ad Hoc Networks, Philadelphia, PA, USA; 2004 Oct 1. p. 66-75.

6. Ahmed SAM, Ariffin SHS, Fisal N. Overview of Wireless Access in Vehicular Environment (WAVE) protocols and standards. Indian Journal of Science and Technology. 2013; 6(7):4994-5001.

7. Sharef BT, Alsaqour RA, Ismail M. Vehicular communication ad hoc routing protocols: A survey. Journal of Network and Computer Applications. 2014; 40:363-96.

8. Iyer A, Kherani A, Rao A et al. Secure V2V communications: Performance impact of computational overheads. In the Proceedings of the Institute of Electrical and Electronics Engineers (IEEE) INFOCOM Workshops; 2008 Apr. p. 1-6.

9. Jerbi M, Senouci SM. Characterizing multi-hop communication in vehicular networks. In the Proceedings of the Institute of Electrical and Electronics Engineers (IEEE) Wireless Communications and Networking Conference WCNC; 2008. p. 3309-13.

10. Girinath DR, Selvan S. A novel cluster based routing algorithm for hybrid mobility model in VANET. International Journal of Computer Applications. 2010; 1(15):35-42.

11. Fonseca A, Vazão T. Applicability of position-based routing for VANET in highways and urban environment. Journal of Network and Computer Applications. 2013; 36(3):961-73. 
12. Mahajan RM, Bang AO. Study of various geographic position based routing protocols in VANETS. In the National Conference on CONVERGENCE; 2015. p. 28.

13. Banerjee R, Bhattacharyya CKr. Cluster based routing algorithm with evenly load distribution for large scale networks. In the Proceedings of the Institute of Electrical and Electronics Engineers (IEEE) International Conference on Computer Communication and Informatics (ICCCI); 2014 Jan. p. 1-6.

14. Gao N, Tang L, LI S et al. A hybrid clustering-based MAC protocol for vehicular ad hoc networks. In the International Workshop on High Mobility Wireless Communications (HMWC), Institute of Electrical and Electronics Engineers (IEEE); 2014. p. 183-7.

15. Vodopivec S, Bešter J, Kos A. A survey on clustering algorithms for vehicular ad-hoc networks. In the 35th International Conference on Telecommunications and Signal Processing (TSP), Institute of Electrical and Electronics Engineers (IEEE); 2012. p. 52-6.

16. LI F, Wang Y. Routing in vehicular ad hoc networks: A survey. Institute of Electrical and Electronics Engineers (IEEE) Vehicular Technology Magazine. 2007; 2(2):12-22.

17. Al-Sultan S, Al-Doori MM, Al-Bayatti AH et al. A comprehensive survey on vehicular Ad Hoc network. Journal of Network and Computer Applications. 2014; 37:380-92.

18. Liu J, Wan J, Wang Q et al. A survey on position-based routing for vehicular ad hoc networks. Télécommunication Systems. 2016 May; 62(1):15-30.

19. Dua A, Kumar N, Bawa S. A systematic review on routing protocols for vehicular ad hoc networks. Vehicular Communications. 2014; 1(1):33-52.

20. Zhan FB, Noon CE. Shortest path algorithms: an evaluation using real road networks. Transportation Science. 1998; 32(1):65-73.

21. Perkins CE, Bhagwat P. Highly dynamic DestinationSequenced Distance-Vector routing (DSDV) for mobile computers. In the Proceedings of the Association for Computing Machinery (ACM) SIGCOMM Computer Communication Review; 1994. p. 234-44.

22. Clausen T, Hansen G, Christensen L et al. The optimized link state routing protocol, evaluation through experiments and simulation. In the Institute of Electrical and Electronics Engineers (IEEE) Symposium on Wireless Personal Mobile Communications; 2001.

23. Pei G, Gerla M, Chen T-W. Fisheye state routing: A routing scheme for ad hoc wireless networks. In Institute of Electrical and Electronics Engineers (IEEE) International Conférence on Communications; 2000. p. 70-4.

24. Roth U. Highly dynamic destination-sequenced distance-vector routing; 2011.

25. Lee S-J, Toh C-K, Gerla M. Performance evaluation of table-driven and on-demand ad hoc routing protocols. In the Proceedings of the Institute of Electrical and Electronics Engineers (IEEE) International Symposium on Personal, Indoor and Mobile Radio Communications (PIMRC); 1999. p. 297-301.

26. Clausen T, Jacquet P. Optimized link state routing protocol (OLSR). Network Working Group; 2003 Oct. p. 4-75.

27. Park VD, Corson MS. A highly adaptive distributed routing algorithm for mobile wireless networks. In the Sixteenth Annual Joint Conference of the Institute of Electrical and Electronics Engineers (IEEE) Computer and Communications Societies, Driving the Information Révolution INFOCOM; 1997. p. 1405-13.

28. Royer EM, Perkins CE. Multicast operation of the adhoc on-demand distance vector routing protocol. In the Proceedings of the 5th annual Association for Computing Machinery (ACM)/ Institute of Electrical and Electronics Engineers (IEEE) International Conference on Mobile Computing and Networking; 1999. p. 207-18.

29. Namboodiri V, Agarwal M, Gao L. A study on the feasibility of mobile gateways for vehicular ad-hoc networks. In the Proceedings of the 1st Association for Computing Machinery (ACM) International Workshop on Vehicular Ad Hoc Networks; 2004. p. 66-75.

30. Johnson DB, Maltz DA. Dynamic source routing in ad hoc wireless networks. In the Mobile Computing, Springer, US; 1996. p. 153-81.

31. Guo S, Yang O, Shu Y. Improving source routing reliability in mobile ad hoc networks. Institute of Electrical and Electronics Engineers (IEEE) Transactions on Parallel and Distributed Systems. 2005; 16(4):362-73.

32. Ratliff S, Dowdell J, Perkins C. Dynamic MANET On-demand (AODVv2) Routing; 2013.

33. Qasim N, Said F, Aghvami H. Mobile ad hoc networks simulations using routing protocols for performance comparisons. In the Proceedings of the World Congress on Engineering; 2008. p. 2-4.

34. Johnson D, Hu Y, Maltz D. The Dynamic Source Routing protocol (DSR) for mobile ad hoc networks for IPv4. Network Working Group; 2007 Feb. p. 5-102.

35. Feeney L M. An energy consumption model for performance analysis of routing protocols for mobile ad hoc networks. Mobile Networks and Applications. 2001; 6(3):239-49.

36. Chakeres ID, Belding-Royer EM. AODV routing protocol implementation design. In the Proceedings of the 24th International Conference on Distributed Computing Systems Workshops, Institute of Electrical and Electronics Engineers (IEEE); 2004. p. 698-703.

37. Chakeres ID, Klein-Berndt L. AODVjr, AODV simplified. In the Proceedings of the Association for Computing Machinery (ACM) SIGMOBILE Mobile Computing and Communications Review. 2002; 6(3):100-1. 
38. Perkins C, Belding-Royer E, Das S. Ad hoc on-demand distance vector (AODV) routing. Network Working Group; 2003 Jul. p. 2-37.

39. Bai PTK, Sundararajan M. Performance efficiency of OLSR and AODV protocols in manets. Indian Journal of Science and Technology. 2015 Jul; 8(14):1-4.

40. Haas ZJ. A new routing protocol for the reconfigurable wireless networks. In the 6th International Conference on Universal Personal Communications Record, Institute of Electrical and Electronics Engineers (IEEE); 1997. p. 562-6.

41. Nikaein N, Bonnet C, Nikaein N. Harp-hybrid ad hoc routing protocol. In the Proceedings of International Symposium on Telecommunications (IST); 2001. p. 56-67.

42. Haas ZJ, Pearlman MR. The performance of query control schemes for the zone routing protocol. Institute of Electrical and Electronics Engineers (IEEE)/ Association for Computing Machinery (ACM) Transactions on Networking (TON). 2001; 9(4):427-38.

43. Haas ZJ, Pearlman MR, Samar P. The Zone Routing Protocol (ZRP) for ad hoc networks [Internet]. 2002 [cited 2002 Jul]. Available from: https://tools.ietf.org/html/draftietf-manet-zone-zrp-04.

44. Liu C, Kaiser J. A survey of mobile ad hoc network routing protocols. University of Ulm, Germany; 2003 Oct. p. 5-35.

45. Malik A, Pandey B. Performance analysis of various data collection schemes used in VANET. Indian Journal of Science and Technology. 2015 Jul; 8(15):1-8.

46. Nikaein N, Labiod $\mathrm{H}$, Bonnet C. DDR: distributed dynamic routing algorithm for mobile ad hoc networks. In the Proceedings of the 1st Association for Computing Machinery (ACM) International Symposium on Mobile ad hoc networking and computing, Institute of Electrical and Electronics Engineers (IEEE) Press; 2000. p. 19-27.

47. Korkmaz G, Ekici E, Özgüner F. An efficient fully adhoc multi-hop broadcast protocol for inter-vehicular communication systems. In Institute of Electrical and Electronics Engineers (IEEE) International Conference on Communications (ICC); 2006. p. 423-8.

48. Khan AA, Stojmenovic I, Zaguia N. Parameterless broadcasting in static to highly mobile wireless ad hoc, sensor and actuator networks. In the 22nd International Conference on Advanced Information Networking and Applications (AINA), Institute of Electrical and Electronics Engineers (IEEE); 2008. p. 620-7.

49. Korkmaz G, Ekici E, Özgüner F et al. Urban multi-hop broadcast protocol for inter-vehicle communication systems. In the Proceedings of the 1st Association for Computing Machinery (ACM) International Workshop on Vehicular Ad Hoc Networks; 2004. p. 76-85.

50. Wu H, Fujimoto R, Guensler R et al. MDDV: a mobility-centric data dissemination algorithm for vehicular networks. In the Proceedings of the 1st Association for Computing
Machinery (ACM) international workshop on Vehicular ad hoc networks; 2004. p. 47-56.

51. Kakarla J, Sathya SS, Laxmi BG et al. A survey on routing protocols and its issues in VANET. International Journal of Computer Applications. 2011 Aug; 28(4):38-44.

52. Sabahi F. The security of vehicular ad hoc networks. In the Third International Conference on Computational Intelligence, Communication Systems and Networks (CICSyN), Institute of Electrical and Electronics Engineers (IEEE); 2011. p. 338-42.

53. Ros FJ, Ruiz PM, Stojmenovic I. Reliable and efficient broadcasting in vehicular ad hoc networks. In the 69th Institute of Electrical and Electronics Engineers (IEEE) Vehicular Technology Conference (VTC), Spring; 2009. p. 1-5.

54. Zeadally S, Hunt R, Chen Y-S et al. Vehicular ad hoc networks (VANETS): status, results, and challenges. Telecommunication Systems. 2012; 50(4):217-41.

55. Allal S, Boudjit S. Geocast routing protocols for VANETs: survey and geometry-driven scheme proposal. Journal of Internet Services and Information Security (JISIS). 2013; 3(1/2):20-36.

56. Bachir A, Benslimane A. A multicast protocol in ad hoc networks inter-vehicle geocast. In the 57th Institute of Electrical and Electronics Engineers (IEEE) Semiannual Vehicular Technology Conference (VTC), Spring; 2003. p. 2456-60.

57. Kihl M, Sichitiu M, Joshi H. Design and evaluation of two geocast protocols for vehicular ad-hoc networks. Journal of Internet Engineering; 2008 Jan 1.p. 1-10.

58. Jerbi M, Beylot A-L, Senouci SM et al. Geo-localized virtual infrastructure for vanets: Design and analysis. In the Conference on Institute of Electrical and Electronics Engineers (IEEE) Global Telecommunications (GLOBECOM); 2008. p. 1-6.

59. Benslimane A, Bachir A. Inter-vehicle geocast protocol supporting non-equipped GPS vehicles. In the Ad-Hoc, Mobile, and Wireless Networks. Springer Berlin Heidelberg; 2003. p. 281-6.

60. Di Felice M, Bedogni L, Bononi L. Group communication on highways: An evaluation study of geocast protocols and applications. Ad Hoc Networks. 2013; 11(3):818-32.

61. Feng W, Elmirghani JMH. Green ICT: Energy efficiency in a motorway model. In the Third International Conference on Next Generation Mobile Applications, Services and Technologies (NGMAST), Institute of Electrical and Electronics Engineers (IEEE); 2009. p. 389-94.

62. Roychowdhury S, Patra C. Geographic adaptive fidelity and geographic energy aware routing in ad hoc routing. In the International Conference on Advances in Computer, Communication Technology and Applications (ACCTA); 2010. p. 309-13.

63. Yu Y, Govindan R, Estrin D. Geographical and energy aware routing: A recursive data dissemination protocol for 
wireless sensor networks. [Internet]. 2001 [cited 2001 Aug 14]. Available from: http://citeseerx.ist.psu.edu/viewdoc/ download?doi=10.1.1.125.7826\&rep=rep1\&type $=$ pdf.

64. Jerbi M, Senouci SM, Doudane YG et al. Geo-localized virtual infrastructure for urban vehicular networks. In the 8th International Conference on Telecommunications (ITS), Institute of Electrical and Electronics Engineers (IEEE); 2008. p. 305-10.

65. Zeadally S, Hunt R, Chen Y-S et al. Vehicular ad hoc networks (VANETS): status, results, and challenges. Telecommunication Systems. 2012; 50(4):217-41.

66. Chen Y-S, Lin Y-W, Lee S-L. A mobicast routing protocol in vehicular ad-hoc networks. Mobile Networks and Applications. 2010; 15(1):20-35.

67. Chen Y-S, Lin Y-W. A mobicast routing protocol with carryand-forward in vehicular ad hoc networks. International Journal of Communication Systems. 2014 ; 27(10):1416-40.

68. Sharef BT, Alsaqour RA, Ismail M. Comparative Study of Variant Position-Based VANET Routing Protocols. Procedia Technology. 2013; 11:532-9.

69. Ko Y-B, Vaidya NH. Location-Aided Routing (LAR) in mobile ad hoc networks. Wireless Networks. 2000; 6(4):307-21.

70. Abbasi IA, Nazir B, Abbasi A et al. A traffic flow-oriented routing protocol for VANETs. EURASIP Journal on Wireless Communications and Networking. 2014; 2014(1):1-14.

71. Lochert C, Hartenstein H, Tian J et al. A routing strategy for vehicular ad hoc networks in city environments. In the Proceedings of the Institute of Electrical and Electronics Engineers (IEEE) Intelligent Vehicles Symposium; 2003. p. 156-61.

72. Seet B-C, Liu G, Lee B-S et al. A-STAR: A mobile ad hoc routing strategy for metropolis vehicular communications. In Networking, Springer Berlin Heidelberg; 2004. p. 98999.

73. Granelli F, Boato G, Kliazovich D. MORA: A movementbased routing algorithm for vehicle ad hoc networks. In the Institute of Electrical and Electronics Engineers (IEEE) Workshop on Automotive Networking and Applications (Auto Net), San Francisco, USA; 2006.

74. Mo Z, Zhu H, Makki K et al. MURU: A multi-hop routing protocol for urban vehicular ad hoc networks. In the Third Annual International Conference on Mobile and Ubiquitous Systems: Networking and Services, Institute of Electrical and Electronics Engineers (IEEE); 2006. p. 1-8.

75. Jerbi M, Senouci S-M, Rasheed T et al. Towards efficient geographic routing in urban vehicular networks. Institute of Electrical and Electronics Engineers (IEEE) Transactions on Vehicular Technology. 2009; 58(9):5048-59.

76. Waluyo AB, Taniar D, Rahayu $\mathrm{W}$ et al. Mobile Peer-to-Peer data dissemination in wireless ad-hoc networks. Information Sciences. 2013; 230:3-20.
77. Sharef BT, Alsaqour RA, Ismail M. Vehicular communication ad hoc routing protocols: A survey. Journal of Network and Computer Applications. 2014; 40:363-96.

78. Zhao J, Cao G. VADD: Vehicle-assisted data delivery in vehicular ad hoc networks. Institute of Electrical and Electronics Engineers (IEEE) Transactions on Vehicular Technology. 2008; 57(3):1910-22.

79. Yu D, Ko Y-B. FFRDV: fastest-ferry routing in DTN-enabled vehicular ad hoc networks. In the 11th International Conference on Advanced Communication Technology (ICACT), Institute of Electrical and Electronics Engineers (IEEE); 2009. p. 1410-14.

80. Burgess J, Gallagher B, Jensen D et al. Max prop: routing for vehicle-based disruption-tolerant networks. In the INFOCOM. 2006. p. 1-11.

81. Soares VNGJ, Rodrigues JJPC, Farahmand F. GeoSpray: A geographic routing protocol for vehicular delay-tolerant networks. Information Fusion. 2014; 15:102-13.

82. Singh V, Dadhich R. Efficient routing by minimizing end to end delay in delay tolerant enabled VANETs. International Bulletin of Mathematical Research. 2015 Mar; 2(1):241-5.

83. Kang H, Ahmed SH, Kim D et al. Routing protocols for vehicular delay tolerant networks: a survey. International Journal of Distributed Sensor Networks. 2015; 2015(4):1-9.

84. Marzak B, Toumi H, Talea M et al. Cluster head selection algorithm in vehicular Ad Hoc networks. In the International Conference on Cloud Technologies and Applications (CloudTech), Institute of Electrical and Electronics Engineers (IEEE); 2015. p. 1-4.

85. Hassanabadi B, Shea C, Zhang L et al. Clustering in vehicular ad hoc networks using affinity propagation. Ad Hoc Networks. 2014; 13:535-48.

86. Daeinabi A, Rahbar AGP, Khademzadeh A. VWCA: An efficient clustering algorithm in vehicular ad hoc networks. Journal of Network and Computer Applications. 2011; 34(1):207-22.

87. Wolny G. Modified DMAC clustering algorithm for VANETs. In the 3rd International Conference on Systems and Networks Communications (ICSNC); 2008. p. 268-73.

88. Goonewardene RT, Ali FH, Stipidis, Elias. Robust mobility adaptive clustering scheme with support for geographic routing for vehicular ad hoc networks. Intelligent Transport Systems (IET). 2009; 3(2):148-58.

89. Koulakezian A. Aspire: Adaptive service provider infrastructure for VANETs [Doctorat thesis]. Canada, University of Toronto; 2011.

90. Dror E, Avin C, Lotker Z. Fast randomized algorithm for hierarchical clustering in vehicular ad-hoc networks. In the 10th IFIP Annual Mediterranean Ad Hoc Networking Workshop (Med-Hoc-Net), Institute of Electrical and Electronics Engineers (IEEE); 2011. p. 1-8. 
91. Basu P, Khan N, Little TDC. A mobility based metric for clustering in mobile ad hoc networks. In the International conference on Distributed computing systems workshop, Institute of Electrical and Electronics Engineers (IEEE); 2001. p. 413-8.

92. Harrabi S, Chainbi W, Ghedira K. A multi-agent approach for routing on vehicular ad-hoc networks. Procedia Computer Science. 2013; 19:578-85.

93. Vodopivec S, Bešter J, Kos A. A survey on clustering algorithms for vehicular ad-hoc networks. In the 35th International Conference on Telecommunications and Signal Processing (TSP), Institute of Electrical and Electronics Engineers (IEEE); 2012. p. 52-6.

94. Wang S-S, Lin Y-S. Performance evaluation of passive clustering based techniques for inter-vehicle communications. In the 19th Annual Wireless and Optical Communications Conference (WOCC), Institute of Electrical and Electronics Engineers (IEEE); 2010. p. 1-5.

95. Morales MMC, Hong CS, Bang Y-C. An adaptable mobility-aware clustering algorithm in vehicular networks. In the 13th Asia-Pacific Network Operations and Management Symposium (APNOMS), Institute of Electrical and Electronics Engineers (IEEE); 2011. p. 1-6.

96. Maslekar N, Boussedjra M, Mouzna J et al. A stable clustering algorithm for efficiency applications in VANETs. In the 7th International Conference on Wireless Communications and Mobile Computing Conference (IWCMC); 2011. p. 1188-93.

97. Ahizoune A, Hafid A, Ben Ali R. A contention-free broadcast protocol for periodic safety messages in vehicular Ad-hoc networks. In the 35th Conference on Local Computer Networks (LCN), Institute of Electrical and Electronics Engineers (IEEE); 2010. p. 48-55.

98. Jaap S, Bechler M, Wolf L. Evaluation of routing protocols for vehicular ad hoc networks in typical road traffic scenarios. In the Proceedings of the 11th EUNICE Open European Summer School on Networked Applications; 2005. p. 584-602.

99. Wei X, Qing-Quan L. Performance evaluation of data disseminations for vehicular ad hoc networks in highway scenarios. The International Archives of the Photogrammetry, Remote Sensing and Spatial Information Sciences, Beijing. 2008; XXXVII(B1):1015-20.

100.Perkins CE. Ad hoc networking. Addison-Wesley Professional; 2008.

101.Vodopivec S, Bešter J, Kos A. A multihoming clustering algorithm for vehicular ad hoc networks. International Journal of Distributed Sensor Networks. 2014 Mar; 2014(4):1-8.

102.Marzak B, El Guemmat K, Talea $M$ et al. Clustering in vehicular ad-hoc network using artificial neural network. International Review on Computers and Software (IRECOS). 2016; 11(6):548-56.

103. Marzak B, Toumi H, Benlahmar E et al. Performance analysis of routing protocols in vehicular ad hoc network. In Advances in Ubiquitous Networking, Springer, Singapore; 2017. p. 31-42. 